\title{
Hybridization and Selective Release of DNA Microarrays
}

N. R. Beer, B. Baker, T. Piggott, S. Maberry, C. M. Hara, J. DeOtte, W. Benett, E. Mukerjee, J. Dzenitis, E. K. Wheeler

December 7, 2011 
This document was prepared as an account of work sponsored by an agency of the United States government. Neither the United States government nor Lawrence Livermore National Security, LLC, nor any of their employees makes any warranty, expressed or implied, or assumes any legal liability or responsibility for the accuracy, completeness, or usefulness of any information, apparatus, product, or process disclosed, or represents that its use would not infringe privately owned rights. Reference herein to any specific commercial product, process, or service by trade name, trademark, manufacturer, or otherwise does not necessarily constitute or imply its endorsement, recommendation, or favoring by the United States government or Lawrence Livermore National Security, LLC. The views and opinions of authors expressed herein do not necessarily state or reflect those of the United States government or Lawrence Livermore National Security, LLC, and shall not be used for advertising or product endorsement purposes.

This work performed under the auspices of the U.S. Department of Energy by Lawrence Livermore National Laboratory under Contract DE-AC52-07NA27344. 


\section{Hybridization and Selective Release of DNA Microarrays}

N. R. Beer, B. Baker, T. Piggott, S. Maberry, C.M. Hara, J. DeOtte, W. Benett, E. Mukerjee, J. Dzenitis, E.K. Wheeler

\section{Project Overview}

DNA microarrays contain sequence specific probes arrayed in distinct spots numbering from 10,000 to over 1,000,000, depending on the platform. This tremendous degree of multiplexing gives microarrays great potential for environmental background sampling, broad-spectrum clinical monitoring, and continuous biological threat detection. In practice, their use in these applications is not common due to limited information content, long processing times, and high cost. Our work focused on characterizing the phenomena of microarray hybridization and selective release that will allow these limitations to be addressed. This will revolutionize the ways that microarrays can be used for LLNL's Global Security missions.

\section{Project Goals}

The goals of this project were two-fold: automated faster hybridizations and selective release of hybridized features. The first study area involves hybridization kinetics and mass-transfer effects. The standard hybridization protocol uses an overnight incubation to achieve the best possible signal for any sample type, as well as for convenience in manual processing. There is potential to significantly shorten this time based on better understanding and control of the rate-limiting processes and knowledge of the progress of the hybridization. In the hybridization work, a custom microarray flow cell was used to manipulate the chemical and thermal environment of the array and autonomously image the changes over time during hybridization.

The second study area is selective release. Microarrays easily generate hybridization patterns and signatures, but there is still an unmet need for methodologies enabling rapid and selective analysis of these patterns and signatures. Detailed analysis of individual spots by subsequent sequencing could potentially yield significant information for rapidly mutating and emerging (or deliberately engineered) pathogens. In the selective release work, optical energy deposition with coherent light quickly provides the thermal energy to single spots to release hybridized DNA. This work leverages LLNL expertise in optics, microfluidics, and bioinformatics.

\section{Relevance to LLNL Mission}

LLNL has ongoing efforts in detection methods against biological terrorism.

The next stage of molecular diagnostics for biological threats is to look much more broadly for emerging threat bio-signatures, such as virulence elements or natural and engineered mutations. This capability is targeted against new natural pandemics and engineered biological warfare agents, while still detecting the full set of known bio-threat agents, to enable prompt countermeasures. 


\section{Accomplishments and Results}

In-situ hybridization - Using a previously designed flow cell for microarrays we were able to successfully demonstrate in-situ hybridization. The scan of the microarray in Figure 1 shows an example of an array hybridized in our flow cell. Because the system was automated both for flow control as well as scanning, we were able to obtain scans of the array during hybridization. During the initial stage of hybridization scans were taken every 15 minutes. After the first hour, images were only obtained every hour. The plots on the right of Figure 1 show the intensity of the features as a function of time for two different locations within the flow cell. The intensity plots are slightly different due to imperfect mixing within the flow cell. The hybridization buffer entered the flow cell from the bottom and resulted in more uniform fluid than the top. One future step is to improve the homogeneity of the solutions in the flow cell by either better flow condition or adding mixing chevrons onto the top surface of the flow cell. Regardless of the differences in location hybridization for this array was complete within 2 hours. More studies are needed to fully evaluate the necessary hybridization time as a function of starting amount of material.

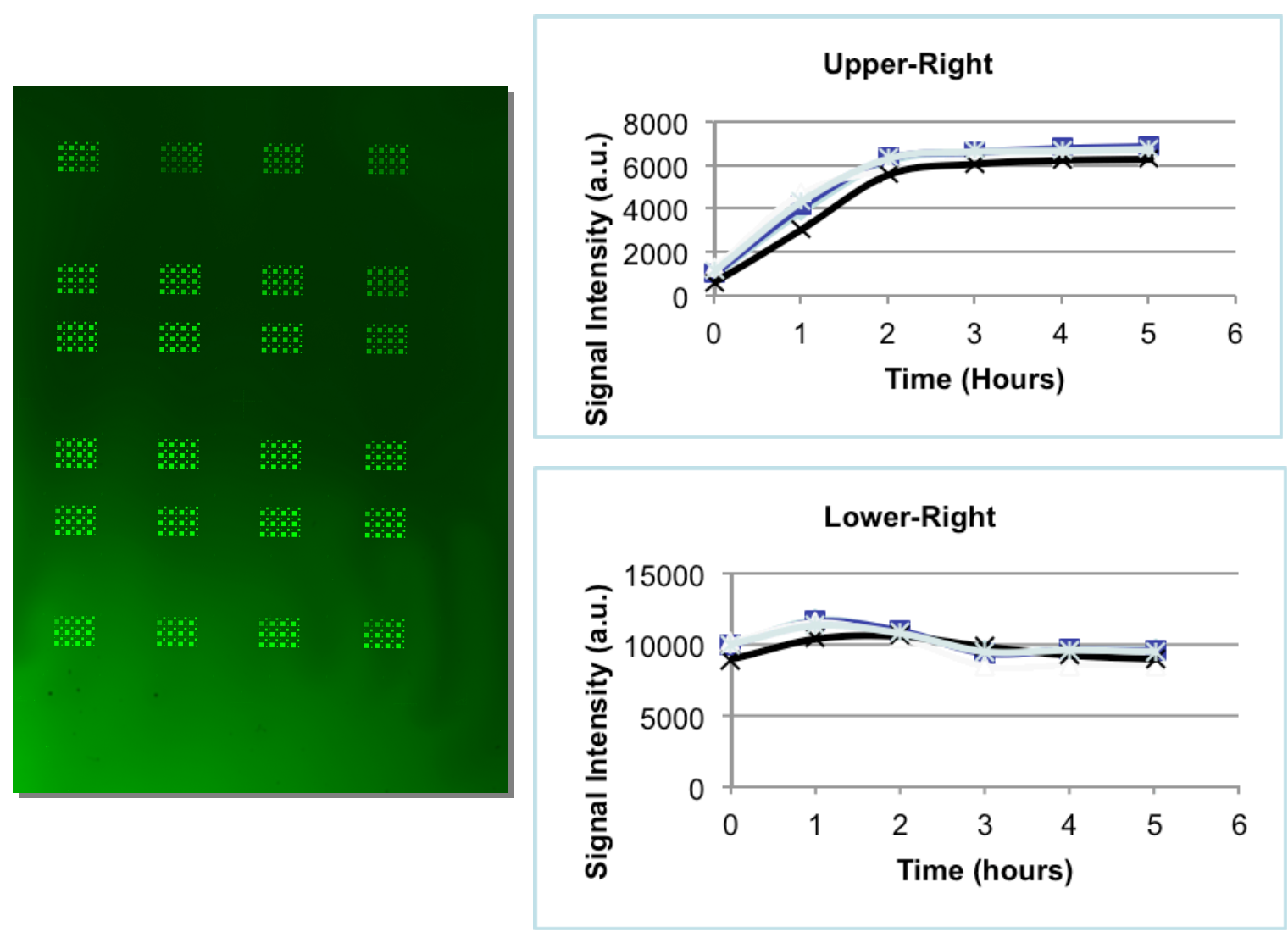

Figure 1: In-situ hybridization of microarray (left) with analysis of hybridization as a function of time. 
Laser Induced DNA Dehybridization (LIDD) - As described above post-processing microarrays can yield important information about anomalous hybridization patterns. However, until recently, the state of the art for selective recovery of a feature of interest involved scraping the surface to recover the DNA. As a result of this LDRD, we recently demonstrated an automated method to selectively release DNA bound to the surface of a microarray and recover the DNA for downstream analysis.

Our approach for selective release used laser heating to elute the DNA of interest and then microfluidics to recover the eluted DNA for analysis. A schematic of our laser induced DNA desorption (LIDD) is shown below.

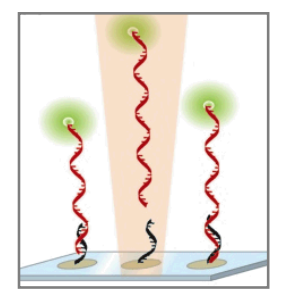

Figure 2: Cartoon of laser induced heating for DNA dehybridization

Lasers provide the ability to selectively direct and focus the coherent light to Abbelimited spot sizes that are ideal for heating aqueous samples with micron-scale features such as the spots on the hybridized microarray surface. A photograph of our LIDD system is shown in Figure 3.

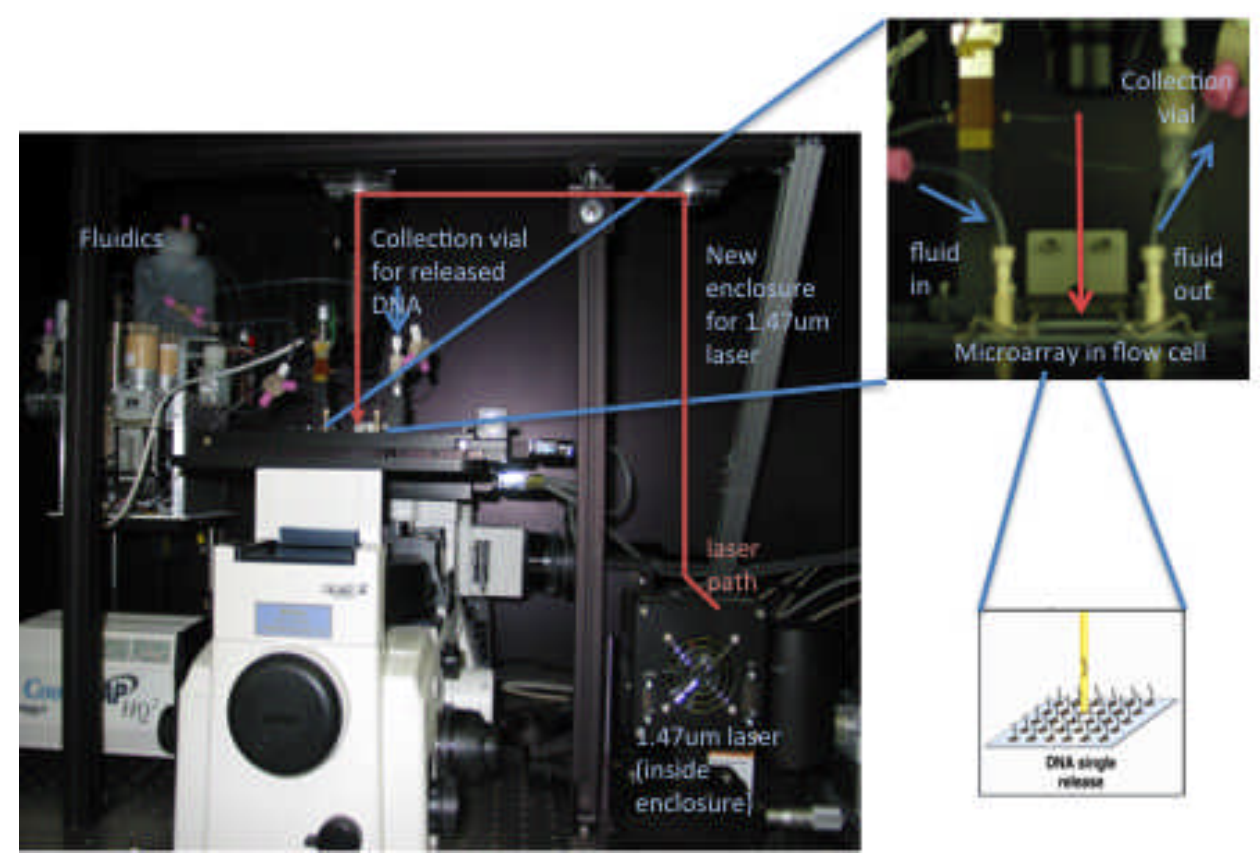

Figure 3: Photograph of LIDD apparatus

In summary, a diode laser at a wavelength of $1.47 \mu \mathrm{m}$ is focused through a lens onto the surface of the microarray. The microarray is enclosed in a flow cell and 
mounted onto a scanning stage on a fluorescence microscope. Flow is controlled with a simple syringe pump. Imaging, flow, stage position and laser firing are all computer controlled.

Firing the laser at targeted features on a microarray containing probes for both the SARS virus and Rhinovirus we have been able to successfully dehybridize DNA. Typical results following dehybridization are shown in Figu re 4. The top two images of Figure 4 show the microarray after hybridization but prior to laser elution. The lower images in Figure 4 show 3 eluted spots (left image) and 30 eluted spots (right image).

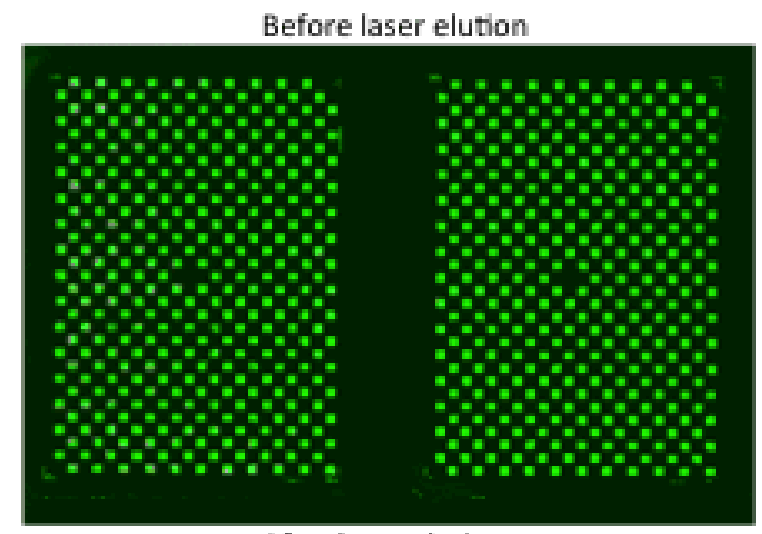

After laser elution

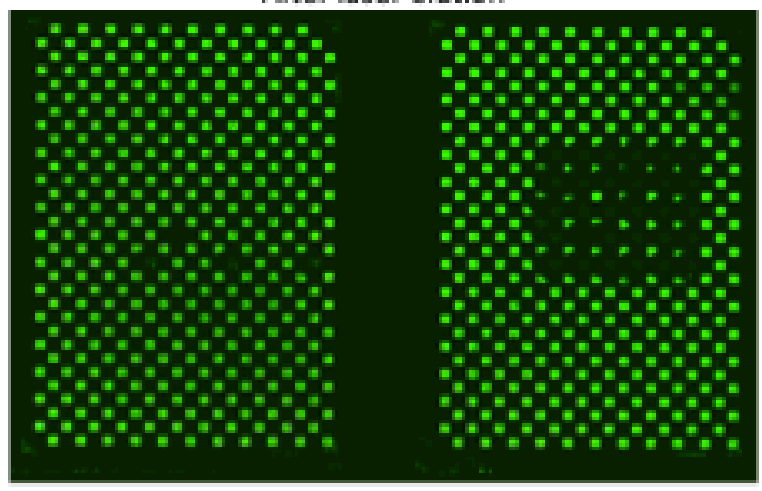

Figure 4: Top image shows the microarray prior to elution; lower images show post laser elution. Lower left shows 3 spots that were subjected to LIDD. Lower right shows an array of 30 eluted spots.

Figure 5 shows line traces of the intensity along the three la ser eluted spots. Prior to elution (top image, Figure 5) all features are fluorescent and the intensities are relatively uniform and high. After laser elution (lower image, Figure 5) features targeted by the laser show a dramatic decrease in intensity where the laser was fired. 


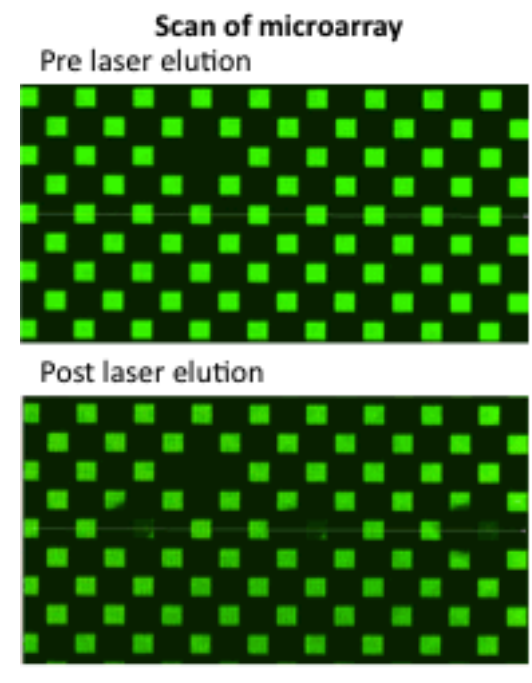

Intensity along line of scanned image

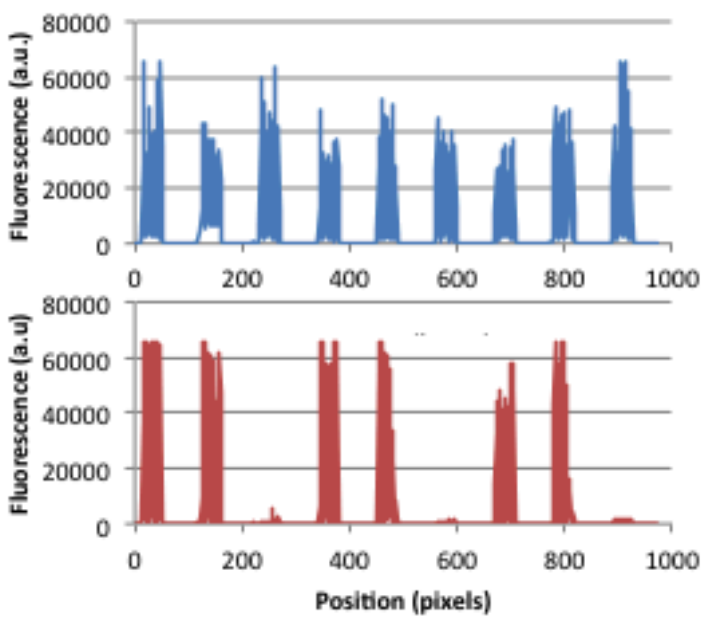

Figure 5: Line traces show significant decreases in intensity at the locations the laser was fired at.

To confirm that we are not just damaging the fluorophore, we collected the eluted DNA and successfully performed PCR assays. If the DNA had been damaged by the laser, then the PCR assay would not have worked. Verifying the viability of the DNA for downstream assays was a key step for future implementation of this technology.

Having demonstrated not only selective feature release but also DNA recovery, we are now well positioned to enable even more widespread use of microarrays. Selectively releasing a feature for downstream sequencing or analysis will potentially enable microarray use in personalized medicine, cancer diagno stics, drug discovery or biosurveillance.

\section{Conclusion}

The successful demonstration of in-situ hybridization and selective release has been demonstrated. Future work could entail reducing spot size for higher density arrays, improving diffusion and mixing within the flow cell, and investigating reductions in starting concentrations to confirm decreased hybridization times.

\section{Acknowledgements}

This work was funded by LDRD 08-ERD-064. This work performed under the auspices of the U.S. Department of Energy by Lawrence Livermore National Laboratory under Contract DE-AC52-07NA27344. 